ACCEPTED MANUSCRIPT

\title{
Oxygen vacancies dynamics in redox-based interfaces: Tailoring the memristive response
}

To cite this article before publication: CRISTIAN FERREYRA et al 2019 J. Phys. D: Appl. Phys. in press https://doi.org/10.1088/1361$\underline{6463 / a b 46 \mathrm{~d} 3}$

\section{Manuscript version: Accepted Manuscript}

Accepted Manuscript is "the version of the article accepted for publication including all changes made as a result of the peer review process, and which may also include the addition to the article by IOP Publishing of a header, an article ID, a cover sheet and/or an 'Accepted Manuscript' watermark, but excluding any other editing, typesetting or other changes made by IOP Publishing and/or its licensors"

This Accepted Manuscript is @ 2019 IOP Publishing Ltd.

During the embargo period (the 12 month period from the publication of the Version of Record of this article), the Accepted Manuscript is fully protected by copyright and cannot be reused or reposted elsewhere.

As the Version of Record of this article is going to be / has been published on a subscription basis, this Accepted Manuscript is available for reuse under a CC BY-NC-ND 3.0 licence after the 12 month embargo period.

After the embargo period, everyone is permitted to use copy and redistribute this article for non-commercial purposes only, provided that they adhere to all the terms of the licence https://creativecommons.org/licences/by-nc-nd/3.0

Although reasonable endeavours have been taken to obtain all necessary permissions from third parties to include their copyrighted content within this article, their full citation and copyright line may not be present in this Accepted Manuscript version. Before using any content from this article, please refer to the Version of Record on IOPscience once published for full citation and copyright details, as permissions will likely be required. All third party content is fully copyright protected, unless specifically stated otherwise in the figure caption in the Version of Record.

View the article online for updates and enhancements. 


\title{
Oxygen vacancies dynamics in redox-based interfaces: Tailoring the memristive response
}

\author{
Cristian Ferreyra ${ }^{1}$, Wilson Román Acevedo ${ }^{1}$, Ralph Gay ${ }^{2}$, Diego Rubi ${ }^{1}$ \\ ${ }^{1}$ GIyA and INN, CNEA, Av. Gral Paz 1499 (1650), San Martín, Buenos Aires, Argentina. \\ ${ }^{2}$ CIC nanoGUNE, Tolosa Hiribidea 76, 20018 Donostia-San Sebastián, Spain.
}

INN, Centro Atómico Baría José Sánchez and Instituto Balseiro, 8400 San Carlos de Bariloche, Argentina.

(Dated: September 12, 2019)

\begin{abstract}
Redox-based memristive devices are among the alternatives for the next generation of non-volatile memories, but also candidates to emulate the behavior of synapses in neuromorphic computing devices. Nowadays it is well established that the motion of oxygen vacancies at the nanoscale is the key mechanism to reversibly switch metal/insulator/metal structures from insulating to conducting, i.e. to accomplish the resistive switching effect. The control of oxygen vacancies dynamics has direct effects on the resistance changes, and therefore on different factors of memristive devices such as switching speed, retention, endurance or energy consumption. Advances in this direction demand not only experimental techniques that allow measuring oxygen vacancies profiles, but also theoretical studies that shed light on the involved mechanisms. Along thesê goals, we analize the oxygen vacancies dynamics in redox interfaces formed when an oxidizable metallic electrode is in contact with the insulating oxide. We show how the transfer of oxygen vacancies can be manipulated by using different electrical stimuli protocols that allow optimizing device figures such as ON/OFF ratio or writing energy dissipation. Analytical expressions for both high and low resistance states are derived in terms of total oxygen vacancies transferred at the interface. Our predictions are validated with experiments performed in $\mathrm{Ti} / \mathrm{La}_{1 / 3} \mathrm{Ca}_{2 / 3} \mathrm{MnO}_{3}$ redox memristive devices.
\end{abstract}

\section{INTRODUCTION}

Memristive devices initially emerged as one of the main alternatives to current flash memory technologies but, more recently, they have been also proposed for $\operatorname{logic}^{1}$ and neuromorphic devices ${ }^{2}$. The physical phenomenon behind memristors is the so-called Resistive Switching (RS) effect, which is the reversible and non-volatile change of the resistance of a metal/insulator/metal structure upon the application of electrical stimulus ${ }^{3-5}$. RS has been ubiquitously found in simple and complex oxides based devices including manganese oxides known as manganites ${ }^{6}$. In these compounds RS is usually of bipolar type, which requires opposite polarities for the electrical stimuli to achieve both SET (high to low resistance, $\mathrm{HR} \rightarrow \mathrm{LR}$ ) and RESET (low to high resistance, LR $\rightarrow$ HR) transitions.

It is well established that interfaces play a key role in many memristive systems (see, for example, Ref. 7 and references therein). Interfacial memristive mechanisms include Schottky barrier modulation due to electromigration of oxygen vacancies ${ }^{8}$, trapping and detrapping of charge carriers by interfacial traps ${ }^{9}$ and the oxidation/reduction of nanoscale interfacial ultrathin layers, formed when oxidizable metals such as Ta, Ti or Al are used as electrodes ${ }^{10,11}$. In the latter case, a thin oxide layer $\left(\mathrm{TaO}_{x}, \mathrm{TiO}_{x}\right.$ or $\left.\mathrm{AlO}_{x}\right)$ of a few nanometers is naturally formed at the interface between the metal electrode and the insulating oxide, and its oxidation/reduction upon electrical cycling drives the memristive behaviour. Moreover, it was shown that it is possible to engineer memristive devices and improve their performance by in- troducing additional layers that regulate ionic exchange between the insulator and the electrode ${ }^{12,13}$. In the case of $\mathrm{Ti} / \mathrm{LCMO}^{14}$, the manganite is spontaneously reduced after the deposition of $\mathrm{Ti}$. The redox reaction is: $\left(\mathrm{La}_{1 / 3}^{3+} \mathrm{Ca}_{2 / 3}^{2+}\right)\left(\mathrm{Mn}_{1 / 3}^{3+} \mathrm{Mn}_{2 / 3}^{4+}\right) \mathrm{O}_{3}^{2-}+\mathrm{Ti} \leftrightarrow$ $\left(\mathrm{La}_{1 / 3}^{3+} \mathrm{Ca}_{2 / 3}^{2+}\right)\left(\mathrm{Mn}_{1 / 3-2 x}^{3+} \mathrm{Mn}_{2 / 3+2 x}^{4+}\right) \mathrm{O}_{3-x}^{2-}+\mathrm{TiO}_{x}$, giving place to a mixed interface $\mathrm{TiO}_{x} / \mathrm{LCMO}_{3-x}$, where the $\mathrm{TiO}_{x}$ layer behaves as a n-type semiconductor and is in contact with the p-type reduced manganite, forming a n$\mathrm{p}$ diode. In these cases, the $\mathrm{RS}$ behavior has been related to a redox process involving the transfer of oxygen ions at the $\mathrm{TiO}_{x} /$ manganite interface ${ }^{10,15}$, while the other interfaces behave as ohmic ${ }^{16,17}$. Moreover, we have recently shown that the redox process is activated after the $\mathrm{n}-\mathrm{p}$ diode is polarized either in direct mode or in inverse mode above breakdown ${ }^{18}$.

The Voltage Enhanced Oxygen Vacancies drift (VEOV) model was originally developed to explain the RS behaviour in single manganites samples ${ }^{8}$ and it was further extended to analyze binary oxides based devices $^{19}$. It has been extensively tested in several memristive Metal-1/Oxide/Metal-2 systems, for oxides ranging from manganites (PCMO, LCMO) and cuprates (YBCO) to binary oxides like $\mathrm{TiO}_{2}$, and metallic electrodes such as $\mathrm{Pt}, \mathrm{Au}, \mathrm{Cu}$ or $\mathrm{Al}^{8,19-23}$.

Recently, the VEOV model has been also adapted to mimic the $\mathrm{RS}$ behavior in $\mathrm{Ti} / \mathrm{LCMO} / \mathrm{Pt}$ samples, where the mixed $\mathrm{TiO}_{x} / \mathrm{LCMO}_{3-x}$ interface dominates the memristive behavior of the device as a consequence of the already described redox process ${ }^{18}$.

With quite a few exceptions ${ }^{22,24,25}$ most of the theoretical studies disregard the connection between oxy- 
gen vacancies dynamics and the manipulation of the attained resistance states. The ability to reversibly control oxygen vacancies profiles should have a straightforward impact on the resistance changes, allowing the improvement of practical devices performance. This can lead, for example, to the optimization of switching speeds or to the minimization of the energy consumption during the writing process. Advances in this direction demand not only experimental techniques that allow measuring oxygen vacancies dynamics ${ }^{26,27}$, but also theoretical studies that shed light on the involved mechanisms. In order to achieve this, we perform a systematic analysis of oxygen vacancies dynamics at redox interfaces and their response to different protocols and stimuli. We show that the simulations indicate how the electrical stimuli can be manipulated to control oxygen vacancies dynamics to optimize memristive figures such as the $\mathrm{ON} / \mathrm{OFF}$ ratio or the energy consumption linked to the RESET process.

In addition, we derive analytical expressions for the attained resistance values in terms of both the total amount of oxygen vacancies transferred along the interface and the applied voltage, which allows the reconstruction of the $\mathrm{R}$ vs $\mathrm{V}$ resistance hysteresis switching loops (HSL).

Our predictions are validated with experiments performed on the $\mathrm{Ti} / \mathrm{LCMO}$ interface, demonstrating the capability of this kind of simulations to understand the physics related to redox memristive processes, paving the way to optimize the electrical response of practical devices.

\section{THE VEOV MODEL REVISITED FOR MIXED REDOX INTERFACES}

In order to be self contained and to clarify notation, we describe here the main assumptions and equations of the $\mathrm{VEOV}^{8,19}$ migration model adapted for the study of $\mathrm{RS}$ in the mixed $\mathrm{TiO}_{x} / \mathrm{LCMO}_{3-x^{28}}$ interface.

The interface is the active region for the RS behavior, and it is modelled as a $1 \mathrm{D}$ chain of $\mathrm{N}=\mathrm{Nl}+\mathrm{Nr}$ total sites, where $N l$ and $N r$ sites are associated to $\mathrm{TiO}_{x}$ and $\mathrm{LCMO}_{3-x}$ layers, respectively. The sites physically represent small domains of nanoscopic dimensions in both sub-oxides with an initial oxygen vacancies concentration corresponding to the pristine state (PS).

We characterize each domain $i$ along the chain by its resistivity $\rho_{i}$ which is a function of the local oxygen vacancies density, $\delta_{i}$. An universal feature of oxides is that their resistivity is dramatically affected by the precise oxygen stoichiometry. LCMO is a complex oxide that behaves as a p-type semiconductor in which oxygen vacancies disrupt Mn-O-Mn bonds with an increase of resistivity. On the other hand, $\mathrm{TiO}_{x}$, behaves as n-type semiconductor in which oxygen vacancies increase the conductivity. As a consequence, we adopt for the first $\mathrm{Nl}$ domains associated to the $\mathrm{TiO}_{x}$ the (most simple) relation between resistivity and oxygen vacancies density:

$$
\rho_{i}^{l}=\rho_{0}^{l}-A_{i} \delta_{i}
$$

where we define $\rho_{0}{ }^{l}$ as the residual resistivity of the left layer for negligible oxygen vacancies concentration $\left(\delta_{i}=\right.$ 0 ) and the coefficient $A_{i}$ is constant, specific of the $\mathrm{TiO}_{x}$ layer.

As the model description is given in terms of oxygen vacancies, we conceive the $\mathrm{TiO}_{x}$ as oxygen vacancies doped $\mathrm{TiO}_{2}$ and therefore $\rho_{0}^{l}$ corresponds to the resistivity of $\mathrm{TiO}_{2}$, (i.e. $x \sim 2$ ).

On the contrary, as the resistivity of the $\mathrm{LCMO}_{3-x}$ layer increases with the presence of oxygen vacancies ${ }^{29}$ we define for sites $i=N l+1, N$ :

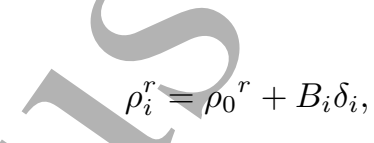

where $\rho_{0}{ }^{r}$ is the residual resistivity of stoichiometric LCMO, and $B_{i}$ is a constant, characteristic of the manganite layer. We notice that both $A_{i}$ and $B_{i}$ can be also taken as smoothly dependent on the site position without affecting the qualitative behaviour of the simulated results.

The total resistance along the interface is computed as $R=c \sum_{i=1}^{N} \rho_{i}$, with the scale factor here taken $c \equiv 1$ for simplicity ${ }^{2}$. Following Eqs.(1) and (2) we obtain

$$
\begin{aligned}
R & =\sum_{i=1}^{N l} \rho_{i}^{l}+\sum_{i=N l+1}^{N} \rho_{i}^{r}, \\
& =R_{s}-\sum_{i=1}^{N l} A_{i} \delta_{i}+\sum_{i=N l+1}^{N} B_{i} \delta_{i},
\end{aligned}
$$

with $R_{s} \equiv N l \rho_{0}{ }^{l}+N r \rho_{0}{ }^{r}$ being the residual resistance of the interface

Given an external stimulus (either current $I(t)$ or voltage $V(t))$ applied to the interface at time t, the oxygen vacancies density at site $i$ is updated for each simulation step according to the probability transfer from site $i$ to a nearest neighbor site $j=i \pm 1$ given by $p_{i j}=\delta_{i}\left(1-\delta_{j}\right) \exp \left(-V_{\alpha}+\Delta V_{i}\right)$. Notice that $p_{i j}$ is proportional to both the oxygen vacancies present at site $i$ and the available occupation at the neighbour site $j^{8}$. In order to restrict the dynamics to the interface region, we take $p_{01}=p_{10}=p_{N N+1}=p_{N+1 N}=0$.

In the Arrhenius factor $\exp \left(-V_{\alpha}+\Delta V_{i}\right), \Delta V_{i}$ is the potential drop at site $i$ defined as $\Delta V_{i}(t)=V_{i}(t)-V_{i-1}(t)$ with $V_{i}(t)=I(t) \rho_{i}=V(t) \rho_{i} / R$. We denote $V_{\alpha}$ the activation energy for oxygen vacancies diffusion in the absence of external stimulus. All the energy scales are taken in units of the thermal energy $k_{B} T$ and we consider $V_{\alpha}=V_{A}$, for sites in the left layer $\left(\mathrm{TiO}_{x}\right)$, and $V_{\alpha}=V_{B}$ for those in the right layer $\left(\mathrm{LCMO}_{3-x}\right)$.

The numerical implementation starts with the input of an initial oxygen vacancies profile at the interface, 
$\delta_{i}(0), \forall i=1 . . N$. We recall that the total amount of oxygen vacancies is assumed to be constant upon electrical cycling. This assumption is valid in real devices if the device toppest layer in contact with the atmosphere behaves as a blocking barrier to both oxygen and moisture. As stated in Ref. 30, capping the top electrode with Au is a suitable strategy for this purpose ${ }^{31}$.

According to standard RS experiments, we chose the stimulus $V(t)$ as a linear ramp following the cycle $0 \rightarrow$ $V_{m 1} \rightarrow-V_{m 2} \rightarrow 0$. At each simulation time step $t_{k}$ we compute the local voltage profile $V_{i}\left(t_{k}\right)$ and the local voltage drops $\Delta V_{i}\left(t_{k}\right)$. Employing the probability rates $p_{i j}$ we compute oxygen vacancies transfer between nearest neighboring sites. Afterwards, the values $\delta_{i}\left(t_{k}\right)$ are updated to a new set of densities $\delta_{i}\left(t_{k+1}\right)$, with which we compute, at time $t_{k+1}$, the local resistivities $\rho_{i}\left(t_{k+1}\right)$, the local voltage drops under the applied voltage $V\left(t_{k+1}\right)$ and finally, from Eq.(3), the total resistance $R\left(t_{k+1}\right)$ to start the next simulation step at $t_{k+1}$.

The oxygen vacancies configuration for the pristine state (PS) has been taken consistently with the experimentally reported low resistance initial state ${ }^{10,18}$, in which the non-stochiometric $\mathrm{TiO}_{x}$ layer $(x<2)$ contributes with a significant conductivity. The partial oxidation of $\mathrm{Ti}$ layer is at expenses of the reduction of a thin LCMO layer that becomes $\mathrm{LCMO}_{3-x}$. This redox process has been clearly identified through spectroscopic characterization by the Jülich group in Ref.10. Taking into account this scenario, the initial oxygen vacancies density at $\mathrm{TiO}_{x}$ allows a significant conductivity for this layer. Additionally, as the resistivity of the $\mathrm{LCMO}_{3-x}$ increases due to the presence of oxygen vacancies, we have chosen a profile for the PS that matches these requirements and is compatible with the (low resistance) initial state of the complete interface (see Fig.1(b)).

In Fig.1(a) we show a typical R vs V Hysteresis Switching Loop (HSL), obtained from the numerical simulations with the VEOV model for a symmetric voltage ramp i.e. $V_{m 1}=\left|V_{m 2}\right|$. Two consecutive cycles are considered in order to show that the first loop displays a slightly erratic response which stabilizes from the 2 nd cycle.

In the experiments reported in Refs.10 and 18, the RESET process takes place for positive stimulus and it is related to the transfer of oxygen vacancies (positive defects) from the $\mathrm{TiO}_{x}$ layer to the $\mathrm{LCMO}_{3-x}$ layer, the first becoming nearly stoichiometric $(x \sim 2)$ and thus highly resistive. At the same time, oxygen vacancies at the $\mathrm{LCMO}_{3-x}$ also contribute to increase the interface resistance. In Fig.1(b), the oxygen vacancies profile associated to the HR state linked to the 1st HSL is shown, and is in complete agreement with the described behaviour.

The SET transition takes place for negative stimulus, when the interface returns to a LR state. The associated oxygen vacancies profile, shown in Fig.1(b), corresponds to the LR state after the completion of the 1st HSL. In this case oxygen vacancies accumulate at the left side of the interface, on the contrary of the initial PS -which indeed has a higher resistance value- where the vacan-
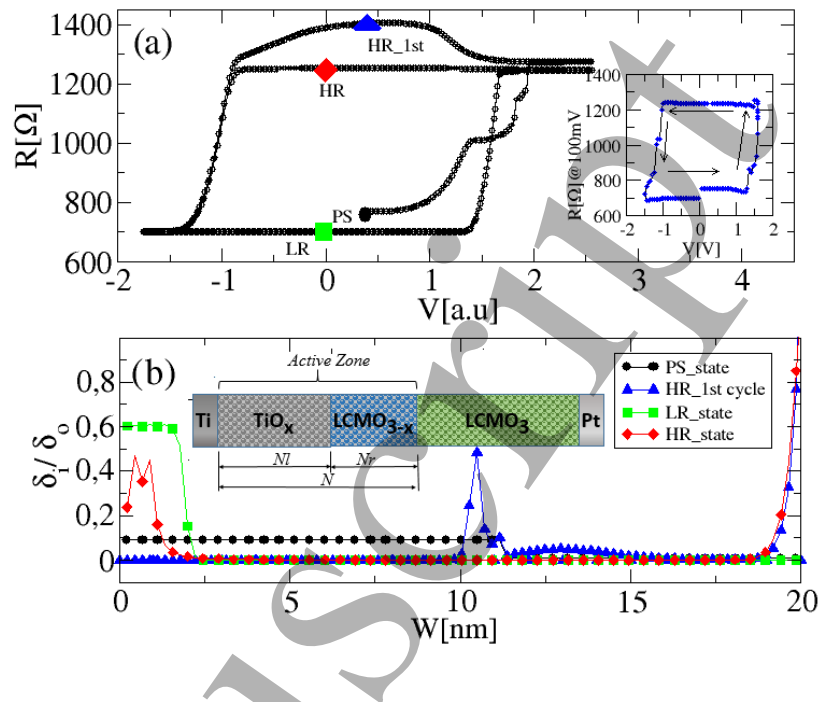

FIG. 1: (a) R vs V (HSL) obtained with the VEOV model for the 1st and 2nd cycles of the voltage protocol $0 \rightarrow V_{m 1} \rightarrow-V_{m 2} \rightarrow 0$. In the simulations we take $\mathrm{W}=20 \mathrm{~nm}, \mathrm{Wl}=11.1 \mathrm{~nm}, V_{A}=8.5, V_{B}=6$. The two latter values are chosen following Refs.19 and 32, which report oxygen vacancies diffusions barrier for $\mathrm{TiO}_{x}$ (up to $2.5 \mathrm{eV})$ and LCMO $(\sim 1.3 \mathrm{eV})$. Inset: Experimental HSL for a single cycle of the voltage protocol, with $V_{m 1}=\left|V_{m 2}\right|=1.8 \mathrm{~V}$. The arrows indicate the circulation of the curve. (b) Normalized oxygen vacancies density profiles along the interface $\delta_{i} / \delta_{0}$, for different resistance states indicated respectively in the HSL's of panel (a). Inset: sketch of the device with the active region considered in the numerical simulations. See text and Ref.28 for details.

cies density is constant. We notice that the difference between PS and LR states is small, and both resistance values remain much lower than the HR state. The resistance of the device is dominated by the contribution of the $\mathrm{LCMO}_{3-x}$ layer ${ }^{18}$, which displays similar oxygen vacancies profiles for both PS and LR states.

Besides the initial behavior of the 1st HSL, the HR and LR states associated to the following cycles are highly repetitive and stable. We notice that the first RESET event can be identified as a forming process, consistently with the work of Herpers et al. ${ }^{16}$ in Ti/PCMO interfaces. Thus the erratic behaviour of the 1st HSL -also exhibited in the 1 st experimental $\mathrm{HSL}^{18}$ - can be attributed to an initial metastable distribution of oxygen vacancies which is washed out during the first electrical cycle, leading upon further cycling to the switching between states with more stable oxygen vacancies configurations. As an example, we show in Fig.1(b) the oxygen vacancies configuration correspondent to the HR state during the 2nd HSL.

The inset of Fig1(a) shows an experimental HSL recorded for the $\mathrm{Ti} / \mathrm{LCMO}$ interface for a complete voltage-stimulated cycle (please see the Supplementary 
Information for details about the device geometry and fabrication procedure). The similarity between the simulated and experimental HSL's is remarkable, demonstrating the ability of the VEOV model to collect the physics of the memristive effect. Notice that as the amount of transferred oxygen vacancies is controlled by the amplitude of the electrical stimuli, different experimental HSLs can be obtained by tuning the voltage (or current) excursions, as it was already discussed in Ref.18.

We also notice that our modelling neglects the presence of self-heating (Joule) effects, which are know to be less relevant in area-dependent memristive effects, as it is our case (evidence about area-dependent memristive behavior is given in the Supplementary Information). In addition, in the experiments presented in this work possible self-heating effects are minimized by using a writing protocol consisting in consecutive single pulses (typical time-widths of 1-10ms) separated by times with no stimuli of (at least) similar magnitude, instead of using continuous voltage ramps. This allows draining the heat produced by Joule effect and avoids a significant temperature rise in the active zone of the device. In Ref. 33 significant Joule heating effects follow the application of continuous triangular voltage ramp with a duration of $100 \mathrm{~ms}$, that is at least one order of magnitude longer that our single pulses. We have also previously shown ${ }^{34}$ that for manganite memristive systems at temperatures close to room temperature, the diffusive contribution to oxygen vacancies migration is a higher order correction to electric field-driven drift of vacancies. These facts indicate that for our system and writing protocol, neglecting self-heating and difussive effects is a good aproximation.

\section{RESISTANCE IN TERMS OF TRANSFERRED OXYGEN VACANCIES}

In this section we go a step further and derive analytical expressions for the resistance values cast in terms of the transferred oxygen vacancies as a function of the applied stimulus.

As in typical experiments, the external electrical stress can be either voltage $V(t)$ or current $I(t)$. For sake of simplicity we consider voltage controlled experiments following the aforementioned protocol, but the following reasoning will be also valid when the stimulus is $I(t)$.

We start from the initial state, correspondent to the configuration depicted in Fig.1 (b), and consistent with the PS. Taking into account Eq.(3) we write

$$
R(0)=R_{s}-A a l_{0}+B a r_{0},
$$

where $R_{s}$ has been previously defined. We denote the left and right initial areas (total number of oxygen vacancies), as $a l_{0} \equiv \sum_{i=1}^{N l} \delta_{i, 0}$ and $a r_{0} \equiv \sum_{i=N l+1}^{N} \delta_{i, 0}$, respectively, with $\delta_{i}(0) \equiv \delta_{i, 0}$ being the oxygen vacancies density at site $i$ for the initial state.

Positive voltages $0<V \leq V_{m 1}$ move vacancies (as positive defects) from the left layer of the interface $\left(\mathrm{TiO}_{x}\right)$ to the right layer $\left(\mathrm{LCMO}_{3-x}\right)$, as we have already described. For each value of $V(t)>0$ it is possible to compute the transferred area of the oxygen vacancies profiles $a^{+}(V(t))$. Taking into account the conservation of the total number of vacancies, we define $a l^{+}(V)=a l_{0}-a^{+}(V)$ and $a r^{+}(V)=a r_{0}+a^{+}(V)$. In this way we can write:

$$
\begin{aligned}
R^{+}(V) & =R_{s}-A a l^{+}(V)+B a r^{+}(V) \\
& =R(0)+(A+B) a^{+}(V),
\end{aligned}
$$

showing that the resistance $R^{+}(V)$ for positive voltages $V(t)$ is determined by the transferréd area $a^{+}(V)$ and sample specific parameters. As $a^{+}$increases, $R^{+}$attains higher values and thus it might be expected that for a sufficiently strong voltage $V_{R} \leq V_{m 1}$, the RESET transition to the HR state takes place, i.e. $R^{+}\left(V_{R}\right) \equiv \mathrm{HR}$. In the next section we will study the oxygen vacancies transfer process in order to analyse different scenarios for the RESET transition. An important issue that will be addressed is whether the RESET takes place for $a^{+}\left(V_{R}\right)=a l_{0}$ (complete transfer of the initial number of $\mathrm{OV})$, or alternatively for $a^{+}\left(V_{R}\right)<a l_{0}$.

For negative voltages, oxygen vacancies move from the right to left side of the interface. Defining $a^{-}(V)$ as the net transferred area for a (negative) voltage $|V| \leq V_{m 2}$, we can write $a l^{-}(V)=a l_{0}-a^{+}\left(V_{R}\right)+a^{-}(V)$ and $a r^{-}(V)=a r_{0}+a^{+}\left(V_{R}\right)-a^{-}(V)$, for the left and right interfaces, respectively. For simplicity we have assumed that once the RESET transition takes place for positive polarities and until the reversal of the voltage polarity, the transfer of vacancies from the right to the left interface is inhibited. This assumption is consistent with the (almost) flat shape of the HSL experimentally observed for this range of voltages (see inset of Fig.1 (a)). Thus we write for $|V| \leq V_{m 2}$,

$$
\begin{aligned}
R^{-}(V) & =R_{s}-A_{a l}^{-}(V)+B a r^{-}(V) \\
& =R_{0}+(A+B)\left\{a^{+}\left(V_{R}\right)-a^{-}(V)\right\} .
\end{aligned}
$$

In analogy with the previous description, we define the SET transition for a negative voltage $\left|V_{S}\right| \leq V_{m 2}$ with an associated transferred area $a^{-}\left(V_{S}\right)$. Therefore, from Eq.(6), the low resistance LR state is $R^{-}\left(V_{S}\right)=R_{0}+$ $(A+B)\left\{a^{+}\left(V_{R}\right)-a^{-}\left(V_{S}\right)\right\} \equiv \mathrm{LR}$.

We can repeat the described procedure for additional cycles of the applied voltage protocol, but as the systematics is essentially the same as the one already detailed, we restrict the explicit description to a single cycle.

From Eqs.(5) and (6) it is possible to reconstruct $R$ for a complete cycle of $V(t)$, i.e. the HSL, once the transferred areas are determined.

Depending on the relation between $a^{+}\left(V_{R}\right)$ and $a^{-}\left(V_{S}\right)$ different scenarios emerge for the LR state. In those cases where $a^{+}\left(V_{R}\right)=a^{-}\left(V_{S}\right)$, the attained LR state results identical to the initial one, see Eq.(6). However, in cases where $a^{+}\left(V_{R}\right) \lesseqgtr a^{-}\left(V_{S}\right)$, LR $\lesseqgtr R_{0}$. These responses have been already observed in the experiments of Ref.18 and generate either close or open HSLs after a complete cycle of the voltage excursion. 
Besides the formal simplicity of Eqs.(5) and (6), the analytical determination of $a^{+}(V)$ and $a^{-}(V)$ is not a trivial task. In the following we summarize the main steps followed to obtain $a^{+}$, and refer the readers to the Appendix for further details. As $V(t)$ is a known function of the (discretized) elapsed time $t \equiv \sum_{k} t_{k}$, the total transferred area can be written as $a^{+}(t)=\sum_{k} a^{+}\left(t_{k}\right)$. To simplify the notation, we denote $a_{k}^{+} \equiv a^{+}\left(t_{k}\right)$. After a lengthy calculation, we can write (see Appendix):

$$
a_{k}^{+}=a_{k}^{+L}+a_{k}^{+N L},
$$

where we define the linear and non linear contributions respectively as:

$$
\begin{array}{r}
a_{k}^{+L}=C_{N l} \delta_{N l}(k) \exp \left(I(k) \rho_{N l}(k)\right)-C_{N l+1} \delta_{N l+1}(k) \\
\exp \left(-I(k) \rho_{N l+1}(k)\right), \\
a_{k}^{+N L}=-\delta_{N l}(k) \delta_{N l+1}(k)\left\{-C_{N l+1} \exp \left(-I(k) \rho_{N l+1}(k)\right)\right. \\
+C_{N l} \exp \left(I(k) \rho_{N l}(k)\right\},
\end{array}
$$

with $I(k)=V(k) / R(k)$, following the adopted convention.

Notice that in the case of current controlled experiments, in which $I(k)$ is known, the above equations indicate that the transferred area $a_{k}^{+}$for the time interyal $t_{k}$ is determined in terms of the density of oxygen vacancies at the two frontier sites of the interface, i.e. $\delta_{N l}(k)$ and $\delta_{N l+1}(k)$, respectively. This is a non-trivial result that could be experimentally tested using oxygen vacancies imaging techniques ${ }^{26,35}$ in current controlled experiments, and should contribute to the design of optimized interfaces for RS experiments.

Equation(8) can be further simplified taking into account that the activation energies satisfy $V_{A}<V_{B}$, which implies $C_{N l}>>C_{N l+1}$. Considering this approximation, the obtained analytical estimates for $a_{k}^{+}$(see Eq.(A12)) enable the determination of the transferred areas as a function of the applied stimulus.

In the Appendix, we also derived estimates for $a_{k}^{-}$(see Eq.(A17)) to compute the transferred area $a^{-}=\sum_{k} a_{k}^{-}$ for the case of negative applied stimulus.

To give a concrete example, we consider current controlled protocols for which the expressions for $a^{+}(I)$ and $a^{-}(I)$ acquire its simplest form, due to the fact that $I(k)$, the current at each time step $t_{k}$, is known. Figure 2 shows the analytical estimates for $a^{+}(I)$ and $a^{-}(I)$ obtained for a current loop $\mathrm{I}(\mathrm{t})=0 \rightarrow I_{m 1} \rightarrow-I_{m 2} \rightarrow 0$. Notice that in this case the conversion from transferred areas to resistance values is trivial following equations analogous to Eqs.(5) and (6). Thus, the analytical reconstruction of the HSL, R vs I, in terms of the applied stimulus is fully accomplished. The analytical estimates, that only consider oxygen vacancies at sites $N l$ and $N l+1$, result almost indistinguishable from the numerical values (see Fig.2) obtained with the VEOV model. In this last case the complete oxygen vacancies profile along the whole interface has to be updated at each simulation step $t_{k}$, which demands an appreciable computational effort.

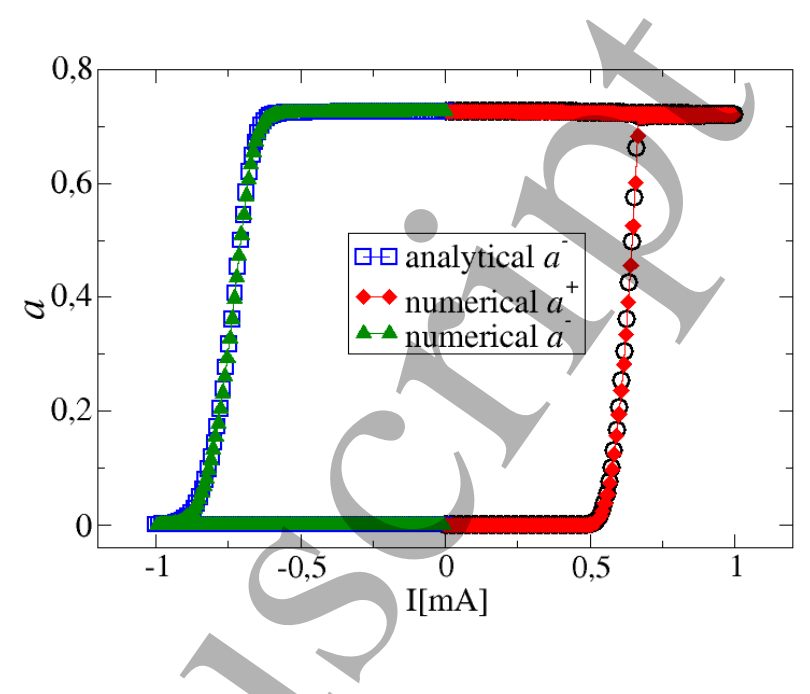

FIG. 2: Adimensional transferred area $a^{+}\left(a^{-}\right)$for a protocol $\mathrm{I}(\mathrm{t})=0 \rightarrow I_{m 1} \rightarrow 0\left(0 \rightarrow-I_{m 2} \rightarrow 0\right)$. The circles (squares) were obtained following the analytical estimates Eq.(A12)) (Eq.(A17)) (normalized in terms of the total density of oxygen vacancies, $\delta_{0}$ ). The diamond

and triangle symbols correspond to the numerical calculations employing the VEOV model simulations.

An important figure of merit is the HR/LR ratio which, from Eqs.(5) and (6), can be expressed as:

$$
\frac{R L-R_{0}}{R H-R_{0}}=\frac{a^{+}\left(V_{R}\right)-a^{-}\left(V_{S}\right)}{a^{+}\left(V_{R}\right)},
$$

taking $R_{0}$ as a reference value.

To give further insight into the area transfer process, in the next section we will analyze the oxygen vacancies dynamics for different electrical protocols. This will allow to determine optimal stimuli protocols, which will be confirmed with experiments peformed on the Ti/LCMO interface.

\section{DYNAMICS OF OXYGEN VACANCIES}

Given the fact that the HR and LR states are essentially determined by areas transferred during RESET and SET transitions, respectively, an interesting and quite unexplored aspect is related to the sensitivity of these processes to the peculiarities of the voltage protocol. Along this goal, in this section we analyse the oxygen vacancies dynamics for different applied stimulus. We concentrate in the RESET process that take place for positive stimulus $V(t)$, but the same analysis can be performed for the SET process.

The starting point is the initial oxygen vacancies configuration, which is shown in both top panels of Fig.3 labeled by $\mathrm{V}=0 \mathrm{~V}$. This distribution defines an initial (adimensional) area $a l_{0}=1$ on the left side of the interface, which we recall corresponds to the $\mathrm{TiO}_{x}$ layer 
$(N l=50$ which gives $\mathrm{Wl}=11.1 \mathrm{~nm}$, see SI material). To analyse the time evolution of this initial density profile, we consider two positive voltage excursions (ramp1 and ramp2) of a linear ramp $0<V \leq V_{m 1}$, which differ in the rising time $T_{i}$. We focus on oxygen vacancies transfer for the 2 nd voltage cycle, to avoid the analysis of the initial transient taking place during the 1st HSL.

In the top panels of Fig.3, we show representative snapshots of the density profiles for selected voltages. The associated transferred areas $a^{+}(V)$ are shown in the lower panels. An important outcome is that the duration of the ramp $T_{i}$ turns out to be a knob that controls whether the transfer is complete or not. Notice that for ramp 1, the transferred area seems to saturate in a value $a_{\text {sat }}^{+} \sim 0.65<a l_{0}=1$, before the completion of the voltage excursion. In addition, a finite amount of vacancies remains in the $\mathrm{TiO}_{x}$ region, consistently with the fact that the complet transfer is not achieved. On the other hand, for ramp 2 (right top panel of Fig.3) the initial area is fully transferred, i.e. $a_{s a t}^{+}=a l_{0}=1$. Indeed this is attained for voltage values lower than $V_{m 1}$ (see the oxygen vacancies profile in the right top panel). From the plateau in each plot of $a^{+}$(lower panels) we can define a saturated area value, $a_{\text {sat }}^{+}$. By doing this we define a plausible criteria to estimate the reset voltage $V_{R}$, as the voltage obtained at the intersection between the horizontal line correspondent to null transfer area with the tangent line at the value $a_{\text {sat }}^{+} / 2$. This is explicitly sketched in both lower panels of Fig. 3. The obtained values of $V_{R}$ are in excellent agreement with the ones extracted from the HSL in the VEOV model simulations.

In the present example the complete transfer of vacancies is attained for ramp 2, with $T_{2}>T_{1}$. We therefore can conclude that, for linear continuous ramps, lower slopes favor the complete transfer from the left to right side of the interface, once the amplitude of the ramp $V_{m 1}$ exceeds a critical voltage necessary to activate the transfer. From the above analysis the onset of the RESET transition is clearly identified with the "first arrival" of the oxygen vacancies front to the right-hand side of the interface $\left(\mathrm{LCMO}_{3-x}\right)$.

Next, we analyze the case of RESET process driven by pulsed voltage ramps, which consist in a series of pulses of increasing amplitude and time width $\Delta T$. Consecutive pulses are separated by $\Delta T$ intervals with no applied voltage, as it is shown in the inset of Fig. 4 (b). This type of voltage protocol is extensively used in RS experiments.

We systematically vary $\Delta T$, leaving the total duration of the ramp constant. In this way, shorter $\Delta T$ are associated to ramps with higher number of pulses. Figures 4(a) and (b) display the correspondent $\mathrm{R}$ vs time and $\mathrm{R}$ vs $\mathrm{V}$ associated to the RESET process, for different $\Delta T$ 's, shown in the legend.

We recall that larger transferred area $a^{+}(V)$ implies larger renmant resistance, as deduced in Eq.(5). It can be observed from the figure that the transferred area is maximized for the shortest pulses, indicating that the OFF/ON (HR/LR) ratio is optimized by accumulating a

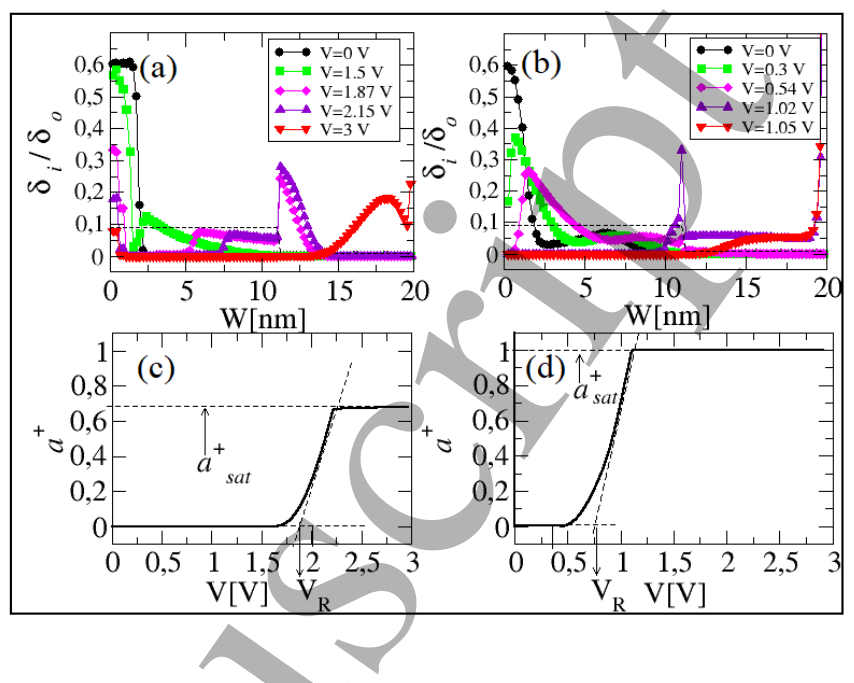

FIG. 3: Top panels: Snapshots of the oxygen vacancies density profiles along the interface for different values of the applied stimulus $\mathrm{V}(\mathrm{t})$, according to two protocols

ramp1 (a) and ramp2 (b), defining a linear ramp $0<V \leq V_{m 1}$, with $V_{m 1}=2.9 \mathrm{~V}$ and rising times $T_{1}=0.1 \mathrm{~s}(\mathrm{ramp} 1)$ and $T_{2}=2.5 \mathrm{~s}(\mathrm{ramp} 2)$, respectively. In dashed black line it is shown the initial OV profile for $t=0, V=0$, which gives an adimensional area $a l_{0}=1$. Lower panels (c), (d): Adimensional

transferred area $a^{+}$for different voltage values shown in the upper panels legends. The RESET voltages $V_{R}$ are estimated following the criteria explained in the text.

higher amount of short pulses rather than a lower amount of wider ones. This non trivial result is experimentally confirmed for the Ti/LCMO interface, as shown in Figs $4(\mathrm{c})$ and (d), which display several RESET processes for voltage pulsed ramps with a fixed total duration of 2.88 $\mathrm{s}$ and different $\Delta T$, ranging between $2-10 \mathrm{~ms}$. It is evident that a higher HR final state is achieved for shorter (2ms) pulses, confirming the prediction derived from the simulations. In addition, the qualitative and quantitative agreement with the numerical predictions is remarkable.

Finally, we address the study of the RESET process for trains of rectangular pulses differing in their timewidths $\Delta T$ and amplitudes $V_{0}$, but keeping the product $V_{0} \times \Delta T=$ constant. We start with an oxygen vacancies profile defining an initial area $a_{l 0}=1$. The RESET process is considered as completed when the initial area is fully transferred (we choose the amplitudes $V_{0}$ in order to satisfy this requirement).

The simulated evolution of $\mathrm{R}$ vs time for the different trains is displayed in Fig.5(a) for $V_{0} \times \Delta T=3 \mathrm{~V}$ ms. It is found that the number of pulses needed to achieve the RESET changes in a non-monotonic way with the amplitude $V_{0}$. This information is indexed in Fig. 5(b), which also displays the electrical energy necessary to complete the RESET process as a function of $V_{0}$. These ener- 


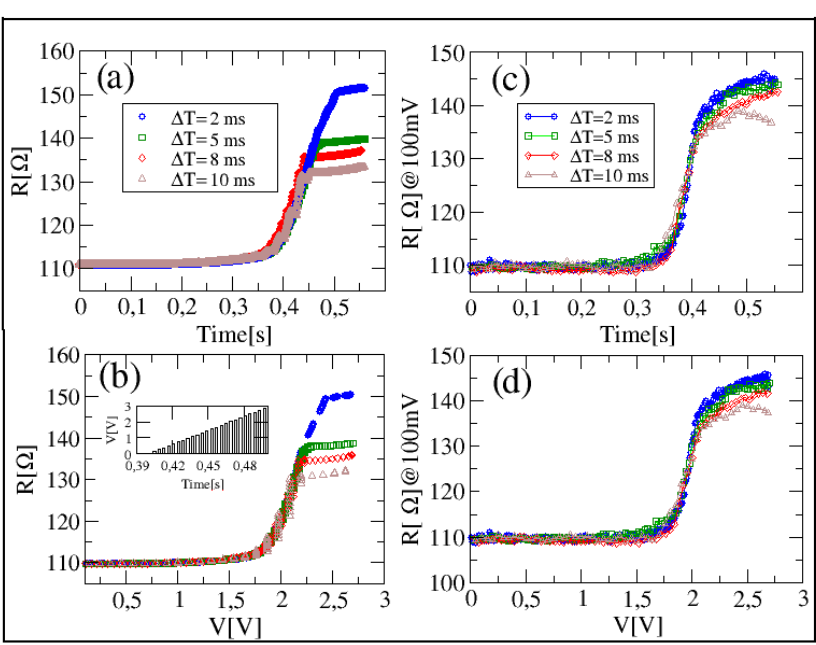

FIG. 4: Left panels: Simulations with the VEOV model of (a) $\mathrm{R}\left(R^{+}\right)$vs time and (b) $\mathrm{R}\left(R^{+}\right)$vs V. Both plots are for a series of pulsed protocols following a linear ramp of amplitude $V_{m}=2.7 \mathrm{~V}$ but differing in the pulse duration $\Delta T$, as shown in the inset of panel (a). A representative ramp is shown in the inset of panel (b). Right panels: Experimental results for (c) remnant resistance $\mathrm{R}$ vs time (c) and R vs V (d). Different curves differ in the time-width of the applied voltage pulses. We notice that these measurements were performed on a device with larger area than in the case of Fig.1(a), leading to lower resistance states

gies were calculated as $U=V_{0}^{2} \sum_{i} \Delta T_{i} / R_{i}$, with $R_{i}$ being the attained resistance value after the application of the $i-t h$ pulse of duration $\Delta T$. From this analysis we conclude that there is a $\left\{\Delta T, V_{0}\right\}$ pair which minimizes the RESET energy. This was indeed verified experimentally for the Ti/LCMO interface, as it is shown in Figs. 5(c) and (d). Pulse trains with $V_{0}$ and $\Delta T$ ranging between [1.8-3] $\mathrm{V}$ and $[1-1.75] \mathrm{ms}$ were tested with the product $\Delta T \times V_{0}=3 \mathrm{~V}$ ms. In the experiment we consider the RESET process as completed, when the relative resistance change after the last applied pulse is below $5 \%$. Again, the number of pulses necessary to complete the RESET process display a non-monotonic dependence with $V_{0}$. To estimate the injected energy during each pulse, we assumed that the resistance increases linearly to its final value during the application of the pulse. Under this aproximation, the trend shown in Figure 5 (d) is in good (qualitative) agreement with the simulated data of Fig.5 (b). An exact determination of experimental RESET energies requires measuring the current transients during the RESET process, which allows tracking nonlinear contributions to oxygen vacancies dynamics ${ }^{36}$.

The present analysis clearly shows that the VEOV simulations appear as a powerful tool to analyze oxygen vacancies dynamics in redox memristive systems and pre- dict optimum writing protocols to increase the efficiency of practical devices.

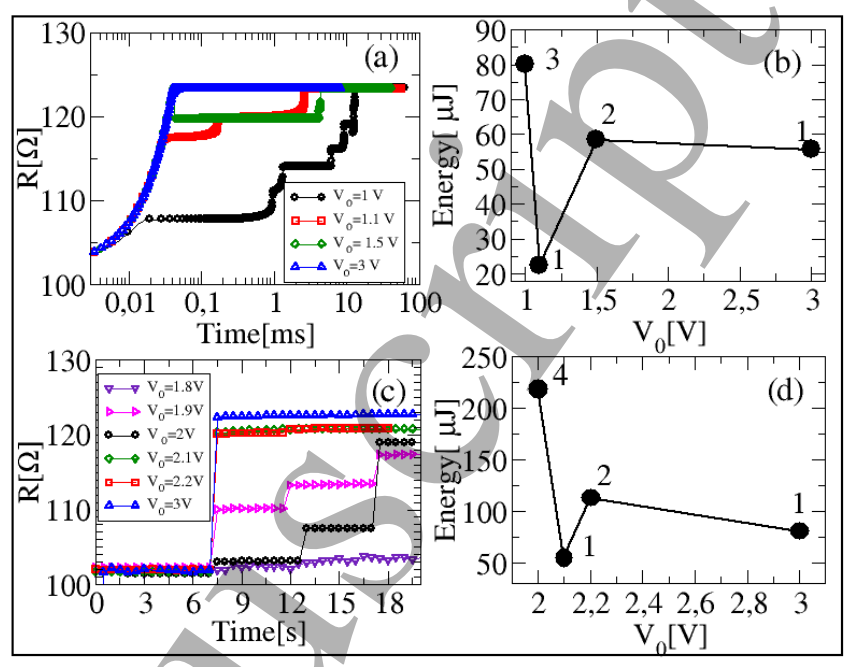

FIG. 5: Top panels: Simulations of R vs time for different trains of rectangular pulses satisfying $V_{0} \times \Delta T \Leftarrow 3 \mathrm{~V}$ ms (a) (see text for details), and Electrical energy injected into the system for a complete RESET process (b). Lower panels: Experimental values of $\mathrm{R}$ ys time for different trains of pulses satisfying $\Delta T \times V_{0}=3 \mathrm{~V} \mathrm{~ms}(\mathrm{c})$, and electrical energy injected into the system for a complete RESET process (d). Panels (b) and (d) have indexed the number of pulses needed for a complete RESET.

\section{CONCLUSIONS}

In summary, we have thoroughly addressed the oxygen vacancies dynamics in redox $\mathrm{p}-\mathrm{n}$ interfaces by using un updated version of the VEOV model. The simulations allow to predict the optimum write protocol to control and enlarge the $\mathrm{ON} / \mathrm{OFF}$ ratio. Our results are relevant not only for memories optimization, but also for neuromorphic computing applications, as the presence of multilevel resistance states allows mimicking the adaptable synaptic weight of brain synapses ${ }^{37}$.

In addition we found the optimum stimuli protocol that minimizes the energy consumption linked to the RESET process. This is also important for the optimization of neuromorphic computing devices aiming to emulate the highly efficient energy consumption of biological systems $^{38}$.

The numerical predictions were fully validated with experiments on the Ti/LCMO memristive interface, demonstrating the power of this type of phenomenological modelling to predict and optimize the behavior of practical memristive devices. We finally notice that the 
stimuli time scale (i.e. pulses time-widht) was chosen in the ms range to facilitate the experimental verification of the simulations predictions. However, we recall that the modelling is not constrained to this time-scale and can also describe and predict oxygen vacancies dynamics at shorter -down to ns- time-scales (see, for instance, how Figure 5(a) describes the evolution of the transient simulated resistances after the application of voltage pulses), which is a key issue to simulate the electrical response of high speed applications.
1 J. Borghetti et al., Nature 464, 873 (2010).

2 S. Yu, Neuro-inspiring computing using resistive synaptic devices, Springer International Publishing (2017).

${ }^{3}$ G. I. Meijer, Science 319, 1625 (2008).

4 A. Sawa, Materials Today 11, 28 (2008).

5 R. Waser, R. Dittmann, G. Staikov and K. Szot, Adv. Mater 21, 2632 (2009).

6 S.Q. Liu, N. J. Wu, and A. Ignatiev, Appl. Phys. Lett. 76, 2749 (2000).

7 I. Valov, Semicond. Sci. Technol. 32093006 (2017).

8 M. J. Rozenberg, M. J. Sánchez, R. Weht, C. Acha, F. Gomez-Marlasca and P. Levy, Phys. Rev. B 81, 115101 (2010).

9 D. S. Shang et al., Phys. Rev. B 73, 245427, (2006).

10 A. Herpers, C. Lenser, C. Park, F. Offi, F. Borgatti, G. Panaccione, S. Menzel, R. Waser and R. Dittmann, Adv. Mat. 26, 2730 (2014).

11 D-Y. Cho, M. Luebben, S. Wiefels, K-S. Lee, and I. Valov, ACS Applied Materials and Interfaces 9, 19287(2017).

12 G. S. Park et al., Nat. Commun. 42382 (2013).

13 J. P. Strachan et al., Beilstein J. Nanotechnol. 4467 (2013).

14 LCMO (PCMO) is the shorthanded notation for $\mathrm{La}_{1 / 3} \mathrm{Ca}_{2 / 3} \mathrm{MnO}_{3}\left(\mathrm{Pr}_{0.48} \mathrm{Ca}_{0.52} \mathrm{MnO}_{3}\right)$.

15 H-S Lee, G. Y. Yeom and H-H Park, J. Phys. D: Appl. Phys. 48 , 465309 (2015).

16 A. Herpers, Electrical characterization of manganite and titanate structures, PhD Thesis, Forschungszentrum Jülich (2014)

17 F. Hossein-Babaei and S.Rahbarpour, Solid-State/Electronics 56, 185 (2011).

18 W. Román Acevedo et al., J. Phys. D: Appl. Phys. 51, 125304(2018).

19 N. Ghenzi, M. J. Sánchez and P. Levy, J. Phys. D: Appl. Phys. 46, 415101 (2013).

20 N. Ghenzi, M. J. Sánchez, F. G. Marlasca, P. Levy and M. J. Rozenberg, J. Appl. Phys. 107, 093719 (2010).

${ }^{21}$ F. G. Marlasca, N. Ghenzi, P. Stoliar, M. J. Sánchez, M. J. Rozenberg, A. G. Leyva and P. Levy, Appl. Phys. Lett. 98, 123502 (2011).

22 N. Ghenzi, M. J. Sánchez, M. J.Rozenberg, P. Stoliar, F. G. Marlasca, D. Rubi and P. Levy, J. Appl. Phys. 111, $084512(2012)$.

${ }^{23}$ D. Rubi, F. Tesler, I. Alposta, A. Kalstein, N. Ghenzi, F. Gomez- Marlasca, M. Rozenberg and P. Levy, App. Phys. Lett. 103163506 (2013).

24 Y.-F Wang et al., Sci. Rep. 5, 10150 (2015).

25 S. Tang et al., Phys. Rev. X 6, 011028(2016).

26 J. H. Jang et al., ACS nano 11, 6942, (2017).

27 S. Bao et al., ACS Appl. Mater. Interfaces 10, 5107 (2018).

28 See Supplementary Information for details.

29 A. Asamitsu et al., Nature 388, 50 (1997).

${ }^{30}$ H. S. Lee et al., Nanoscale 15, 6444 (2015).

$31 \mathrm{Au}$ capping is the strategy we have followed for the de- vices presented in this work to experimentally validate the simulations results. Please see the Supplementary Information for details about the devices geometry and fabrication procedure.

32 T. Nagata, M. Haemori, Y. Yamashita, H. Yoshikawa, Y. Iwashita, K. Kobayashi and T. Chikyow, Appl. Phys. Lett. 99, 223517 (2011).

33 L. Yao et al., Nature Commun. 8, 14544 (2017).

34 P. Stoliar et al., J. Phys. D: Appl. Phys. 47, 435304 (2014).

35 M. Setvin et al, Phys. Rev. B 91, 195403 (2015)

36 A. Marchewka, B. Roésgen, K. Skaja, H. Du, C.-L. Jia, J. Mayer, V. Rana, R. Waser, S. Menzel, Adv. Func. Elect. 2, 1500233 (2016)

37 D. Kuzum et al., Nanotechnology 24, 382001 (2013).

38 P.A. Merolla et al., Science 345, 668 (2014).

\section{ACKNOWLEGMENTS}

We acknowledge support from CNEA, UNCuyo (06/C455) and ANPCyT (PICT2014-1382, PICT20160867, PICT2017-1836). We acknowledge L. Hueso who allowed accessing the nanoGUNE nanofabrication facilities and P. Levy for supporting this collaboration.

\section{Appendix A: Analytical estimates for the transferred areas}

In this Appendix we derive the expressions for the transferred area $a_{k}^{+}$from the left to the right side of the interface, in terms of the linear and nonlinear contributions, Eq.(8).

We start by defining the rate of oxygen vacancies variation between neighbours sites $i-1, i$ and $i+1$ as:

$$
\begin{aligned}
\Delta_{i}(k)= & \left(p_{i-1, i}(k)+p_{i+1, i}(k)\right)- \\
& -\left(p_{i, i-1}(k)+p_{i, i+1}(k)\right),
\end{aligned}
$$

with

$$
p_{i, j}(k)=C_{i} \delta_{i}(k)\left(1-\delta_{j}(k)\right) \exp \left(I(k) \rho_{i}(k)\right)
$$

and $C_{i}=\exp \left(-V_{\alpha}\right)$, already introduced in Sec.II of the main text. In the following we consider $C_{N l}=\exp \left(-V_{A}\right)$ and $C_{N l+1}=\exp -\left(V_{B}\right)$. 
Employing Eq.(A1), we write the transferred area (total number of transferred vacancies) $a_{k}^{+}$as:

$$
\begin{aligned}
a_{k}^{+} \equiv & \sum_{i=N l+1}^{N} \Delta_{i}(k)=\sum_{i=N l+1}^{N} \Delta_{i}^{L}(k)+\sum_{i=N l+1}^{N} \Delta_{i}^{N L}(k)= \\
& =a_{k}^{+L}+a_{k}^{+N L}
\end{aligned}
$$

where we have defined:

$$
\begin{aligned}
\Delta_{i}^{L}(k)= & C_{i-1} \delta_{i-1}(k) \exp \left(I(k) \rho_{i-1}\right)+ \\
& +C_{i+1} \delta_{i+1}(k) \exp \left(-I(k) \rho_{i+1}\right)- \\
& -C_{i}\left[\delta_{i}(k) \exp \left(-I(k) \rho_{i}\right)+\delta_{i}(k) \exp \left(I(k) \rho_{i}\right)\right]
\end{aligned}
$$

and

$$
\begin{aligned}
\Delta i^{N L}(k)= & -C_{i-1} \delta_{i-1}(k) \delta_{i} \exp \left(I(k) \rho_{i-1}\right)- \\
& -C_{i+1} \delta_{i+1}(k) \delta_{i}(k) \exp \left(-I(k) \rho_{i+1}\right) \\
& +C_{i}\left[\delta_{i}(k) \delta_{i-1}(k) \exp \left(-I(k) \rho_{i}\right)+\right. \\
& \left.+\delta_{i}(k) \delta_{i+1}(k) \exp \left(I(k) \rho_{i}\right)\right]
\end{aligned}
$$

Performing the sumations in Eq.(A3) and accounting for the boundary condition $C_{N+1}=0$, we get

$$
\begin{aligned}
& \begin{aligned}
\sum_{i=N l+1}^{N} \Delta_{i}^{L}(k)= & C_{N l} \delta_{N l}(k) \exp \left(I(k) \rho_{N l}\right)= \\
& -C_{N l+1} \delta_{N l+1}(k) \exp \left(-I(k) \rho_{N l+1}\right) \\
& \\
\text { and } & \left.-C_{N l+1} \exp \left(-I(k) \rho_{N l+1}\right)\right) .
\end{aligned} \\
& \sum_{i=N l+1}^{N} \Delta_{i}^{N L}(k)=-\delta_{N l}(k) \delta_{N l+1}(k)\left(C_{N_{l}} \exp \left(I(k) \rho_{N_{l}}\right)-\right. \\
&
\end{aligned}
$$

The linear term $a_{k}^{+L}$ has been written as the sum of two contributions,

defined as:

$$
a_{k}^{+L} \equiv P(k)-Q(k)
$$

$P(k)=C_{N l} \delta_{N l}(k) \exp \left(I(k) \rho_{N l}(k)\right)$, $Q(k)=C_{N l+1} \delta_{N l+1}(k) \exp \left(-I(k) \rho_{N l+1}(k)\right)$.
Analogously, we write the nonlinear term as:

$$
a_{k}^{+N L} \equiv S(k)-T(k)
$$

with

$$
\begin{aligned}
& S(k)=C_{N l+1} \delta_{N l}(k) \delta_{N l+1}(k) \exp \left(-I(k) \rho_{N l+1}(k)\right), \\
& \left.T(k)=C_{N l} \delta_{N l}(k) \delta_{N l+1}(k) \exp \left(I(k) \rho_{N l}(k)\right)\right) .
\end{aligned}
$$

Notice that for current controlled experiments in which the current $I(k)$ is known by input, the tansferred areas at each time interval $t_{k}$ only depend on vacancies densities and resistivities at the sites $N l$ and $N l+1$, respectively.

As we already mentioned in Sec.II, the activation energies for oxygen vacancies diffusion satisfy $V_{A}<V_{B}$ and thus $C_{N l+1} \ll<C_{N l}$. Therefore, we can safely approximate:

$$
a_{k}^{+}=a_{k}^{+L}+a_{k}^{+N L} \approx P(k)-T(k) .
$$

This equation can be updated for the next time interval $t_{k+1}$ employing

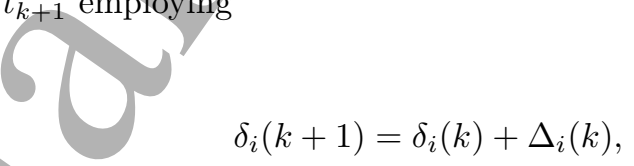

for $i=N l-1, N l$ and $N l+1$, respectively.

From Eq.(A2) and Eq.(A1) we write after a straightforward algebra:

$$
\begin{aligned}
\Delta_{N l}(k) & =C_{N l} \delta_{N l}(k)\left[-2 \cosh \left(I(k) \rho_{l}(k)\right)+\right. \\
& +\delta_{N l+1}(k) \exp \left(I(k) \rho_{N l}(k)\right)+ \\
& \left.+\delta_{N l-1}(k) \exp \left(-I(k) \rho_{N l}(k)\right)\right]+ \\
& +\left(1-\delta_{N l}(k)\right) \\
& {\left[C_{N l+1} \delta_{N l+1}(k) \exp \left(-I(k) \rho_{N l+1}(k)\right)+\right.} \\
& \left.+C_{N l-1} \delta_{N l-1}(k) \exp \left(I(k) \rho_{N l-1}(k)\right)\right] .
\end{aligned}
$$

Performing the sustitution $k \rightarrow k+1$, replacing Eq.(A13) in $P(k)$ (Eq.(A9)), and taking into account Eq.(1) in the main text, we obtain:

$$
\begin{aligned}
P(k+1)= & C_{N l}\left[\delta_{N l}(k)+\Delta_{N l}(k)\right] \\
& \exp \left(I ( k + 1 ) \left(\rho_{0}^{l}-A\left(\delta_{N l}(k)+\Delta_{N l}(k)\right) .\right.\right.
\end{aligned}
$$

In a similar way we derive, after updating $T(k)$ in Eq.(A11),

$$
\begin{aligned}
T(k+1)= & C_{N l}\left[\delta_{N l}(k)+\Delta_{N l}(k)\right]\left[\left(\delta_{N l+1}(k)+\Delta_{N l+1}(k)\right]\right. \\
& \exp \left(I(k+1)\left(\rho_{0}^{l}-A\left(\delta_{N l}(k)+\Delta_{N l}(k)\right)\right) .\right.
\end{aligned}
$$


Employing these two last equations we compute $a_{k+1}^{+} \approx P(k+1)-T(k+1)$.

Following the described prescription iteratively, the total transferred area $a^{+}$after an elapsed time $t_{+}=\sum_{k} t_{k}$, can be computed under the present assumptions.

The same procedure can be applied to compute $a^{-}$ for negative electrical estimulus. Assuming that the current protocol $0 \rightarrow I_{m 1} \rightarrow 0$ is completed for a time $T_{+}=\sum_{k}^{K_{+}} t_{k}$, the initial condition for the negative current protocol $0 \rightarrow-I_{m 2} \rightarrow 0$ should be taken as the oxygen vacancies profile at time $T_{+}$, i.e. $\delta_{i}\left(K_{+}\right)$.
To avoid repetition we give below the final expression, valid for $k>K_{+}$:

$$
\begin{aligned}
a_{k}^{-}= & C_{N_{l}} \delta_{N_{l}}(k) \exp \left(I(k) \rho_{N_{l}}\right)- \\
& -C_{N_{l}+1} \delta_{N_{l}+1}(k) \exp \left(-I(k) \rho_{N_{l}+1}\right)+ \\
& +\delta_{N_{l}}(k) \delta_{N_{l}+1}(k)\left(C_{N_{l}+1} \exp \left(-I(k) \rho_{N_{l}+1}\right)-\right. \\
& \left.\left.-C_{N_{l}} \exp (I(k)) \rho_{N_{l}}\right)\right) .
\end{aligned}
$$

